Estudios Pedagógicos XXXIX, Número Especial 1: 85-101, 2013

INVESTIGACIONES

\title{
Desarrollo de habilidades de observación en estudiantes de pedagogía: Resultados de una intervención piloto basada en el uso de la Videoteca de Buenas Prácticas Docentes*
}

\author{
Developing observation skills in pre-service teachers: Results of a pilot intervention \\ based on the use of the Video Library of Good Teaching Practices
}
Desenvolvimento das habilidades de observação nos estudantes de pedagogia: Resultados de uma intervenção experimental fundamentada na utilização da Videoteca Das Boas Praticas Docentes

\author{
Magdalena Müller ${ }^{a}$, Elisa Calcagni $^{b}$, Valeska Grau $^{c}$, David D. Preiss ${ }^{d}$, Paulo Volante $^{e}$ \\ ${ }^{a}$ Pontificia Universidad Católica de Chile. Telf.: 02/ 23545356. Correo electrónico: mbmuller@uc.cl \\ ${ }^{b}$ Pontificia Universidad Católica de Chile. Telf.: 02/ 23541744. Correo electrónico: mecalcag@uc.cl \\ ${ }^{\mathrm{c}}$ Pontificia Universidad de Chile. Telf.: 02/ 23547279. Correo electrónico: vgaru@uc.cl \\ ${ }^{\mathrm{d}}$ Pontificia Universidad Católica de Chile. Telf.: 02/ 23544635. Correo electrónico: davidpreiss@uc.cl \\ ${ }^{\mathrm{e} P o n t i f i c i a}$ Universidad de Chile. Telf.: 02/ 23545377. Correo electrónico: pvolante@uc.cl
}

\begin{abstract}
RESUMEN
Uno de los aspectos claves de la formación inicial de profesores es la participación de los estudiantes en experiencias pedagógicas en contextos reales. Estas experiencias suelen ser limitadas y parcialmente mediadas por los profesores de los programas de formación. El presente artículo se enmarca en el desarrollo de una videoteca docente, cuyo uso en la formación inicial pretende aumentar la exposición mediada de los estudiantes a prácticas reales. En este contexto, se presenta el diseño, implementación y evaluación de un modelo pedagógico basado en evidencias internacionales sobre video formación. Específicamente, se buscó desarrollar en los estudiantes la capacidad de observación y razonamiento basado en conocimientos. Se aplicó un protocolo de observación de video, y se condujo un análisis de contenido. Los resultados muestran avances de los participantes en su capacidad de focalización en el triángulo estudiantes-profesor-contenido, y en la modalidad de observación, pasando de descripciones generales a específicas, e interpretaciones.
\end{abstract}

Palabras Clave: formación inicial docente, videoteca, video de clases, modalidades de observación, modelo de observación mediada.

\footnotetext{
ABSTRACT

* Los autores agradecen al Ministerio de Educación y, específicamente, al Centro de Perfeccionamiento, Experimentación e Investigaciones Pedagógicas (CPEIP) el acceso a los videos usados en la construcción de la Videoteca de Buenas Prácticas Docentes. Adicionalmente, agradecen a FONDEF de CONICYT el financiamiento que hizo este estudio posible (Proyecto FONDEF D09I1063. Generación de Videoteca de prácticas ejemplares para la formación inicial y continua de profesores en Chile). Magdalena Müller realizó este trabajo como parte de sus estudios conducentes al grado de doctora en Psicología en la Escuela de Psicología de la Pontificia Universidad Católica de Chile. Valeska Grau y David Preiss son también miembros del Centro de Estudios de Políticas y Prácticas en Educación (Proyecto CIE01-CONICYT).
} 
A key aspect of pre-service teachers training is students' participation in real-life educational contexts. However, students' access to these experiences is limited and training programs face practical limitations to fully mediate these experiences. This article comes from a research project aimed at developing an online repository of classroom videos. The web platform, named Videoteca de Buenas Prácticas Docentes, is expected to enhance students' access to mediated observation of real-life classrooms. The present study introduces the design, implementation and evaluation of a teaching model supported on international training experiences. The specific goal was to develop observation skills and knowledge based reasoning among students. A pre and post video observation task was carried out, and data were processed using content analysis. Results show advances in participants' ability to observe and reflect on classroom videos, focusing on the student-teacher-content triangle, and improving their observation stance from general description to specific ones, and also interpretations.

Key words: pre-service teacher education, video-library, classroom video, observation stance, mediated observation model

\section{RESUMO}

Um dos aspectos chave da formação inicial dos professores é a participação dos estudantes em experiências pedagógicas em contextos reais. Estas experiências geralmente são limitadas e parcialmente mediadas pelos professores do programa de formação. O presente artigo tem como marco o desenvolvimento de uma biblioteca docente, enquanto o seu uso na formação inicial tem como objetivo aumentar a exposição guiada dos estudantes à práticas reais. Neste contexto apresenta-se a formulação, implementação e avaliação de um modelo pedagógico, fundamentado em evidencias internacionais sobre vídeos formação. Especificamente, procuro-se desenvolver nos estudantes a capacidade de observação e raciocínio baseado nos conhecimentos. Se aplicou um protocolo de observação de vídeo e se dirigiu uma análise de conteúdo. Os resultados mostram avances dos participantes na sua capacidade de focalização no triângulo estudantesprofessor-conteúdo, e na modalidade de observação, passando de descrições gerais até as específicas, e interpretações.

Palavras chave: videoteca, vídeo formação, modalidades de observação, protocolo de observação, modelo de observação mediada.

\section{INTRODUCCIÓN}

Uno de los desafíos de la Formación Inicial de Profesores (FI) es conectar la formación disciplinar y didáctica con la práctica educativa concreta, ofreciendo oportunidades de vínculo entre teoría y práctica en contextos reales para el desarrollo de habilidades pedagógicas (Darling-Hammond y Hammerness, 2005). Esto es un desafío ya que con frecuencia, el desarrollo de dichas habilidades se focaliza en las experiencias de campo de los estudiantes, ámbito que en general está más alejado del control de los programas de FI (Grossman y Mc Donald, 2008). Estas dificultades para mediar las experiencias que los estudiantes tienen en sus campos de práctica se traducen en una falta de coherencia entre las oportunidades de aprendizaje ofrecidas en los cursos universitarios y las que tienen en su práctica en las escuelas.

$\mathrm{Si}$ bien las experiencias directas de enseñanza son el elemento con mayor impacto en la adquisición de habilidades pedagógicas, existen algunas alternativas que los programas de formación pueden incorporar en sus cursos para acercar la realidad de las escuelas a las aulas universitarias (Darling-Hammond y Hammerness, 2005). La incorporación de planificaciones, evaluaciones y tareas de los alumnos, entre otros, son materiales valiosos que promueven el desarrollo de habilidades analíticas y permiten a los estudiantes relacionar lo que aprenden en los cursos con la sala de clases (Ball y Cohen, 1999). Por otra parte, existen alternativas como por ejemplo las metodologías de microenseñanza, donde los estudiantes se enseñan entre sí (Fernández, 2010), los estudios de 
clase (Sims y Walsh, 2009), los estudios de caso utilizando videos de clase como parte de la metodología en el aula (Eilam y Poyas, 2006), y los estudios de caso a través de foros y plataformas web (Koc et al., 2009).

En este contexto, el presente estudio pretende contribuir al desarrollo de iniciativas que permitan aumentar la exposición mediada de los estudiantes a prácticas en contextos reales. Esto, a través del uso de una videoteca de prácticas docentes y la propuesta del diseño, implementación y evaluación de un modelo de intervención para el uso de videos en formación inicial.

\section{2. $\quad$ ANTECEDENTES TEÓRICOS Y EMPÍRICOS}

\subsection{GENERACIÓN DE UNA VIDEOTECA DE BUENAS PRÁCTICAS}

La generación de una videoteca como una herramienta para la difusión de buenas prácticas pedagógicas surge como una iniciativa de académicos de la Facultad de Educación y la Escuela de Psicología de la Pontificia Universidad Católica de Chile (UC) con financiamiento de un proyecto FONDEF del área TICs-EDU ${ }^{1}$. El recurso consiste en una plataforma web que opera como una biblioteca de videoclips de clases que recogen prácticas ejemplares. La versión inicial reúne videos de profesores y profesoras en las áreas de matemática y lenguaje en segundo ciclo básico $\left(5^{\circ}\right.$ a $\left.8^{\circ}\right)$. Los clips provienen de clases grabadas en el contexto de Docentemás, sistema chileno de evaluación del desempeño profesional docente, y del programa de Asignación de Excelencia Pedagógica (AEP), programa voluntario para la acreditación del desempeño docente (Manzi et al., 2001). Si bien Docentemás y AEP usan la filmación como parte de portafolios de evaluación de desempeño docente, los videos de estos programas han servido como insumo para estudios que buscan caracterizar las prácticas instruccionales en nuestro país (Preiss, 2010, 2009; Preiss et al., 2011; Radovic y Preiss, 2010). En este contexto, esta iniciativa pretende complementar estos usos dando una utilidad formativa a los miles de videos de clases que Chile ha acumulado a partir de estas evaluaciones.

El proyecto que da origen a la Videoteca tiene dos objetivos. En primer lugar, busca generar un modelo conceptual que permita seleccionar prácticas ejemplares, y con ello construir una videoteca digital que dé acceso a los profesionales de la educación a material de video para la formación inicial y el perfeccionamiento docente. En segundo lugar, se busca generar modelos de formación docente inicial y continua utilizando la Videoteca, que permitan probar la utilidad pedagógica de la plataforma así como generar pilotos de perfeccionamiento docente basados en el uso de videos y la observación de clases. En relación con el primer objetivo, el equipo académico desarrolló un Modelo de Buenas Prácticas Docentes (MBP) basado en Marco para la Buena Enseñanza y en literatura especializada. El Modelo incluye Estrategias de Enseñanza que, de acuerdo a la literatura, contribuyen sólidamente al aprendizaje (Alexander, 2008; Anderson et al., 2001; Echevarria y Short, 2004; Hiebert y Stigler, 2000; Milicic et al., 2008; Preiss y Sternberg, 2010; Sternberg y Grigorenko, 2003; Sternberg y Horvath, 1995). También

\footnotetext{
${ }^{1}$ Proyecto FONDEF D09I1063. Generación de Videoteca de prácticas ejemplares para la formación inicial y continua de profesores en Chile.
} 
contempla dimensiones como el Desarrollo de Habilidades de Pensamiento, tomando como referencia la Taxonomía de Bloom, y el fomento de un Ambiente Propicio para el Aprendizaje, que considera la dimensión socio-emocional de la dinámica de clase. Este MBP se transformó en un esquema de códigos que, junto con los contenidos curriculares de lenguaje y matemáticas en segundo ciclo, dio lugar a un proceso de análisis de 1.482 videos de clase (604 de matemática y 878 de lenguaje). Las filmaciones provenían de profesores cuya Evaluación Docente había resultado evaluada en el nivel Destacado, así como de profesores reconocidos por AEP. Los videos fueron codificados por un equipo en cada subsector, compuesto de una profesora experta en el área junto con una psicóloga. Los índices de confiabilidad inter-juez mostraron buenos niveles de coincidencia (81\%). A partir del análisis de los videos se seleccionaron clips de clases que representaran prácticas ejemplares. Además de la selección de clips, el equipo del proyecto desarrolló un Manual de uso que contiene un dossier de lecturas relevantes acerca del uso de videos en el perfeccionamiento docente, junto con guías de ejercicios de observación de videos.

Durante los años 2011 y 2012, la Videoteca Docente estuvo en proceso de construcción y funcionó en una versión piloto cuyo acceso estaba restringido a los integrantes del proyecto, así como a los participantes de las diversas condiciones de pilotaje. Como parte de este pilotaje, se realizaron estudios respecto del uso de tags apropiados como palabras de búsqueda; la usabilidad de la plataforma web con sus diferentes funcionalidades; y una evaluación de la satisfacción con los materiales de apoyo realizada por usuarios y expertos. Cabe destacar que los sujetos consultados reportaron altos niveles de satisfacción respecto de la Videoteca y sus recursos $(95,64 \%)$. A partir del año 2013, el acceso ha sido abierto a profesionales relacionados con el mundo de la educación, quienes pueden registrarse como usuarios. ${ }^{2}$ Con esto, se espera conformar una comunidad de usuarios que utilice los videos y otros recursos de aprendizaje disponibles, como los foros y fichas de lectura.

Tal como se especificaba más arriba, el segundo objetivo de la Videoteca Docente es desarrollar metodologías de formación basadas en video. Esperamos que estas metodologías respondan a la necesidad de aumentar la exposición de los estudiantes de FI a experiencias pedagógicas en contextos reales. De esta forma, se pretende ofrecer nuevas herramientas y modelos de trabajo para la FI. En este contexto, en la Facultad de Educación de la UC se ha realizado un piloto en Formación Inicial. Para diseñar este piloto, se realizó una revisión de la investigación sobre el uso de video de clases como recurso para la formación docente, tanto inicial como continua. A continuación se da cuenta de los principales aportes que emergieron de la revisión de la literatura en el ámbito, y luego se presenta el modelo de intervención elaborado a partir de ésta.

\subsection{VIDEOS DE CLASE COMO RECURSOS DE APRENDIZAJE PARA PROFESORES}

Los videos de sala de clases, o segmentos de ellos, han sido utilizados crecientemente como recursos para traer evidencias de situaciones de enseñanzaaprendizaje a la FI (Tochon, 2007). Al examinar los motivos del aumento del uso de videos, se observa que una de las razones es la masificación y abaratamiento de las tecnologías de grabación y edición de videos. Otra razón es que la grabación de lo que

\footnotetext{
${ }^{2}$ La dirección de la Videoteca es www.videotecadocente.cl
} 
sucede en el aula permite un acceso veraz y directo a sucesos de aula (Schwartz \& Hartman, 2007). Esto ofrece a los participantes de la situación de enseñanza en FI acceder a evidencia que brinda una base común para la discusión. Junto con lo anterior, la investigación internacional ha acumulado a lo largo de las últimas dos décadas numerosa evidencia del efecto positivo que tienen diversas metodologías de formación inicial y continua que utilizan el video de clases como un recurso para el aprendizaje (ver por ejemplo, Borko et al., 2011; van Es y Sherin, 2008).

La observación de videos, de acuerdo con Tochon (2007), reporta ser de mayor efectividad para el aprendizaje cuando es orientada a objetivos y focos claros, en lugar de realizarse sin una orientación específica (ver también Santagata y Angelici, 2010). Acorde con esto, las experiencias y estudios que reportan el uso de videos para la formación docente, ya sea inicial o continua, han adoptado distintos objetivos de aprendizaje:

1) Introducir y modelar metodologías de enseñanza específicas (Kucan et al., 2009; Borko et al., 2008; Borko et al., 2011; Zhang et al., 2011).

2) Desarrollar el conocimiento de la didáctica de una disciplina, como es el caso del Estudio de Clases, desarrollado originalmente en Japón (Lewis, 2002; Perry y Lewis, 2009; Sims y Walsh, 2009).

3) Desarrollar habilidades de observación y reflexión docente. Esto se refiere a la atención selectiva hacia elementos relevantes dentro de la complejidad del aula, así como la capacidad de activar conocimientos atingentes para analizar lo observado (Santagata y Angelici, 2010; Eilam y Poyas, 2006; Sherin y Han, 2004; van Es y Sherin, 2008).

Este tercer objetivo de aprendizaje del uso de videos es el que sirve como referente para la generación del modelo de intervención de este estudio, por lo que se profundizará en las habilidades que implica. Las habilidades de observación, entendiendo como observación la atención selectiva hacia lo relevante, han sido consideradas críticas para la formación docente (Berliner, 1994), dado que se ha visto que los profesores expertos atienden evidencias del aprendizaje de los estudiantes para tomar decisiones pedagógicas. Esto redundaría en opciones de enseñanza más efectivas, al responder a las necesidades de los alumnos y su nivel de comprensión. Los novatos, en tanto, atenderían más a aspectos más superficiales, como la planificación de clase. De esta forma, Santagata y Angelici (2010) afirman que si bien no es razonable esperar que los estudiantes adquieran habilidades de experto a partir de la FI, es relevante que los futuros docentes sepan reconocer y aprender dichas habilidades que favorecen el aprendizaje continuo de docentes y estudiantes a partir de la observación en la práctica.

La reflexión, en tanto, se refiere a la capacidad de examinar la evidencia observada en la práctica, para ganar entendimiento de la situación y generar mejoras ahí donde sean necesarias. Según van Es y Sherin (2008), la literatura del área apunta a complementar las habilidades de qué observar con la capacidad de reflexionar, enfatizando el cómo se observa. Así, sería relevante la capacidad de reconocer lo que sucede en la situación de enseñanza-aprendizaje, y considerarlo en forma interpretativa. Esto, en lugar de evaluar lo observado sin haberlo examinado detenidamente, o compararlo con el "deber ser", que se ha visto que es una tendencia en los profesores a la hora de observar. Otro aspecto relevante en el cómo observar es el nivel de articulación que tengan las observaciones e interpretaciones. En esta línea, Davis (2006) distingue entre reflexiones productivas e improductivas. En las reflexiones productivas los expertos 
serían capaces de reflexionar en forma analítica, relacionando los distintos elementos relevantes de la situación de enseñanza-aprendizaje. Esta forma de reflexión se caracterizaría por ofrecer evidencia que sustente las interpretaciones, cuestionar los supuestos y ofrecer alternativas de acción.

En relación con el constructo de reflexión basado en conocimiento, se ha definido como principal variable la modalidad de observación (Seidel et al., 2011; Sherin y van Es, 2009). En ésta, se distinguen al menos tres niveles (Sherin van Es, 2009):

1) El nivel de descripción, centrado en dar cuenta de lo sucedido en el video de clases;

2) El nivel de evaluación, que implica emitir juicios de valor respecto de la calidad de las interacciones observadas; y

3) El nivel de interpretación, que genera inferencias y relaciones a partir de los elementos observados.

La modalidad de observación ha sido evaluada en los docentes en formación y en ejercicio a partir de discusiones grupales y entrevistas individuales orales, escritas o mediante plataformas digitales. Ésta ha sido abordada mediante dos enfoques diferentes, el primero de ellos trata los datos en forma analítica, segmentando las ideas emitidas por el/la docente y evaluando la frecuencia o proporción en que se observan las distintas modalidades (ver por ejemplo Sherin y van Es, 2009). El segundo enfoque aborda las observaciones sintéticamente, asignando puntajes según la presencia de los distintos niveles de reflexión (Seidel et al., 2011). Estos autores califican las observaciones en forma ordinal, atribuyendo 0 puntos a las descripciones, 1 punto a la combinación de descripción y evaluación, 2 puntos a la combinación de descripción y explicación, y un máximo de 3 puntos a la presencia de las tres modalidades. Este método permite calificar las reflexiones de los sujetos y compararlos.

Sintetizando ambos componentes, van Es y Sherin (2008) articulan las habilidades de observación y reflexión en el concepto de visión profesional docente. El concepto fue acuñado por Goodwin (1994) y llevado al campo docente por Sherin (2007). En la docencia, la visión profesional consiste en la capacidad de atender elementos relevantes de la sala de clases, y activar conocimientos disciplinares y pedagógicos pertinentes para tomar decisiones pedagógicas. Más específicamente, las autoras han propuesto, acorde con las reformas educativas en Estados Unidos, focalizar la atención y razonamiento de los docentes en el pensamiento matemático de los estudiantes, y el seguimiento que hacen de este pensamiento en la sala de clases (Sherin y van Es, 2009).

\subsection{MODELO PARA LA VIDEOFORMACIÓN}

A partir de los antecedentes teóricos y empíricos presentados se llevó a cabo un modelo de intervención centrado en el desarrollo de la visión profesional docente (Sherin, 2007) en los estudiantes de pedagogía. La propuesta general de van Es y Sherin (2009) en torno al desarrollo de la reflexión docente fue complementada con el enfoque del triángulo instruccional, que plantea que la práctica de la enseñanza implica poner en juego los conocimientos y habilidades profesionales para hacer más productivas las interacciones entre tres elementos principales: el estudiante, el profesor o profesora y el contenido (Cohen et al., 2003).

Con esta base se desarrolló un modelo de formación que estableció como focos de observación: el pensamiento de los estudiantes, el contenido y su didáctica y las 
interacciones de aula. Para cada uno de estos focos, el equipo FONDEF elaboró fichas de trabajo que sintetizan la literatura actual en torno a estos temas y formulan una serie de preguntas para incentivar el diálogo y la reflexión. Por otra parte, para el desarrollo de las modalidades de observación se diseñaron, además de las fichas, guías de trabajo que apuntan a la suspensión de los juicios sin evidencia al observar, y al desarrollo de la capacidad de generar descripciones e interpretaciones basadas en evidencia específica (Hammer y Van Zee, 2006; Borko et al., 2008).

\section{OBJETIVOS Y PREGUNTAS DE INVESTIGACIÓN}

En síntesis, los antecedentes relacionados con la evidencia empírica disponible indican los efectos positivos del análisis de videos en el aprendizaje de los profesores. En lo referido a la reflexión docente, se reportan dos componentes principales en las habilidades de observación que se ponen en juego al analizar videos: i) la atención selectiva, y ii) el razonamiento basado en conocimiento (Sherin \& van Es, 2009; van Es y Sherin, 2008). Ambos componentes afectan el qué se releva en una observación y el cómo se interpreta la experiencia observada. Sin embargo, se sabe menos acerca de cómo desarrollar estas habilidades en la trayectoria formativa de los profesores en su formación inicial en Chile. Es por eso que el objetivo general de este estudio piloto es implementar y evaluar experiencias de práctica virtual en la formación inicial de profesores a través del uso de la plataforma de videoteca de prácticas docentes. Así, las preguntas de investigación que guían el estudio son:

1. ¿Cómo influir desde la formación inicial en las habilidades de observación de los estudiantes?

2. ¿Qué observan los estudiantes de pedagogía (foco) y cómo la intervención usando una plataforma virtual de videos de clase impacta en esta dimensión?

3. ¿Cómo observan los estudiantes de pedagogía (modalidad de observación) y cómo la intervención usando una plataforma de prácticas virtuales impacta en esta dimensión?

\section{MÉTODO}

Con el fin de responder a las preguntas del estudio se plantea un estudio exploratorio cuyo objetivo es describir el diseño, implementación y evaluación de un modelo para el desarrollo de habilidades de análisis de videos de clase a través de una plataforma virtual.

\subsection{PARTICIPANTES}

La muestra estuvo compuesta por 16 estudiantes de Pedagogía en Educación General Básica que estaban cursando su práctica profesional. Esta práctica, de un semestre, es la última instancia de su formación profesional y consiste en asistir todos los días de la semana en la jornada de la mañana a establecimientos educacionales seleccionados por la Facultad de Educación. Se optó por implementar el estudio piloto en esta instancia, ya que adicionalmente a su jornada en los colegios, los estudiantes una vez a la semana se reúnen en talleres de supervisión de práctica en la Universidad y este 
espacio tiene por objetivo generar reflexiones pedagógicas basadas en las experiencias de los estudiantes en sus respectivas salas de clases. Pareció una oportunidad entonces el desarrollar habilidades para observar y analizar prácticas pedagógicas en esta instancia. Estos talleres son dirigidos por un(a) supervisor(a) de la Facultad de Educación quien está a cargo de coordinar, acompañar y evaluar las actividades programadas en la práctica. La intervención piloto se hizo en 5 grupos de práctica. El promedio de estudiantes asignados a cada supervisora fue de 4 estudiantes, en un rango de 2 a 7 estudiantes por grupo. Las supervisoras que participaron en la intervención tienen en promedio 7 años de experiencia de supervisión en la Universidad. Fueron seleccionadas por su disponibilidad para dedicar un tiempo de 10 horas semanales adicionales a su labor como supervisoras para capacitarse y diseñar cada sesión de intervención.

\subsection{PROCEDIMIENTO}

Antes de comenzar el semestre académico se conformó un equipo de tarea compuesto por las cinco supervisoras capacitadas, la coordinadora de prácticas del Programa de Educación General Básica y un miembro del equipo FONDEF con el fin de diseñar las sesiones a trabajar en los talleres de la práctica profesional. Tomando como base los principios y materiales del modelo de intervención presentado en los antecedentes, se diseñó una forma de integrar el desarrollo de habilidades de observación focalizada a los objetivos del taller de prácticas. Para esto el equipo de tarea se reunió semanalmente un mes antes de la intervención, instancia que se mantuvo en el transcurso de la implementación. Así se construyó un programa de ocho sesiones de uso de la plataforma que considerara un desarrollo gradual de las modalidades de observación: descripción, interpretación y evaluación, intencionándose en cada sesión un foco de observación: i) el contenido y su didáctica, ii) las interacciones de aula, iii) el pensamiento de los estudiantes. Los contenidos de cada sesión se iban ajustando después de su implementación, considerando las experiencias de cada supervisora en su respectivo grupo.

Para responder a las preguntas 2 y 3 de este estudio se aplicó un instrumento al inicio y al final del semestre para evaluar las habilidades de observación de videos de los estudiantes participantes del piloto. Específicamente, el instrumento de observación de video que se analiza en este estudio consiste en una serie de preguntas abiertas que son respondidas por escrito por los estudiantes, luego de observar un clip de una clase de matemáticas que no habían visto previamente. El clip dura aproximadamente 8 minutos, y fue exhibido dos veces a los estudiantes, para luego entregarles las preguntas de observación. Éstas contemplaban una pregunta inicial que apuntaba a un registro de lo observado en el video sin mayores directrices, cuyas respuestas fueron analizadas para efectos de este estudio. A esto se suman cuatro peguntas más específicas acerca de la profesora, los estudiantes y propuestas de alternativas para la enseñanza. Se consideró que la primera pregunta, menos restringida en su formulación, ofrece la información más relevante acerca de qué y cómo observan los estudiantes al mirar un video de clases. Las respuestas recogidas por escrito fueron digitadas en computador para su posterior análisis. Este tipo de instrumentos ha sido utilizado en investigaciones previas sobre análisis de videos en formación inicial de profesores (Santagata y Angelici, 2010; Eilam y Poyas, 2006; Sherin y Han, 2004; van Es y Sherin, 2008). La aplicación se hizo en cada uno de los grupos de práctica por separado, tanto en el pre como en el post test. 
Los participantes fueron informados de los propósitos del estudio y firmaron una carta de consentimiento de participación, donde accedieron al uso de los datos producidos a la vez que fue comprometido el resguardo la confidencialidad y anonimato respecto de los participantes.

\subsection{ANÁLISIS DE DATOS}

Considerando las preguntas de investigación planteadas en este estudio en relación al qué y cómo miran los estudiantes, se reportarán los análisis de los datos recolectados a partir del instrumento de observación de un video de clases de matemática de segundo ciclo. Tomando como referente algunas de las dimensiones de análisis propuestas por Sherin y van Es (2009), se elaboró un esquema de códigos para cada componente: a) focos de observación (qué se mira), y b) las modalidades de observación (cómo se mira).

\subsubsection{Análisis de los focos de observación}

Considerando como antecedente la definición de Cohen, Raudenbush y Ball (2003) en relación a la importancia de potenciar las interacciones de los elementos del triángulo instruccional para el aprendizaje de los estudiantes, se decidió usar esas tres categorías de codificación para analizar los focos de observación. Así, para analizar el foco de observación de los estudiantes se codificaron las respuestas considerando en qué fijan su atención los estudiantes al observar el clip: estudiante, profesor y contenido. La codificación se hizo en dos niveles: analítico y holístico.

A nivel analítico, se descompuso el registro escrito de los estudiantes en unidades de ideas que equivalían a una oración (van Es y Sherin, 2008). Se analizó cada oración identificando qué elementos del triángulo instruccional se mencionaban. Luego, considerando el número total de unidades de ideas se calculó el porcentaje de éstas que refería a cada elemento. Si bien este análisis permite distinguir los focos de observación de los estudiantes, no informa acerca del nivel de integración con que los estudiantes perciben estos elementos. Es por eso que a nivel holístico se generó una rúbrica de 4 niveles:

1) considera sólo un elemento del triángulo instruccional,

2) integra al profesor y contenido en su registro sin incluir al estudiante, o los dos elementos mencionados no están suficientemente integrados,

3) incluye al estudiante en relación con el profesor o los contenidos, en forma explícita o describe conductas que se puede inferir que son de los alumnos utilizando evidencia del video; y

4) aparecen explícitamente los tres elementos bien articulados.

Para evaluar el grado de confiabilidad en la codificación, se realizaron pruebas de confiabilidad inter-juez entre 2 codificadores obteniéndose un acuerdo de $70 \%$ en cuanto a los niveles de integración de los elementos del triángulo instruccional en la observación. Los desacuerdos fueron discutidos, llegándose a un consenso en todos los casos. 


\subsubsection{Análisis de las modalidades de observación}

Para el análisis de las modalidades de observación se usaron como referentes las modalidades descritas en la literatura: descriptiva, interpretativa y evaluativa (Sherin y van Es, 2009; Seidel et al., 2011). Al describir el estudiante da cuenta de características observables de la actividad del video, al interpretar incluye en su registro inferencias acerca de lo que vio, y al evaluar expresa juicios acerca de la calidad de lo que observa en el video clip. Al analizar los textos, se encontraron patrones de respuesta emergentes que se incorporaron en el plan de análisis en un esquema de códigos de seis categorías:

1) descripción general,

2) descripción detallada y relevante,

3) interpretación con descripción,

4) evaluación sin descripción,

5) evaluación con descripción; y

6) descripción, con interpretación y evaluación.

Los números de cada categoría son nominales y no tienen un valor ordinal. Los datos fueron codificados en forma holística, asignando una categoría a cada respuesta. En caso de detectarse más de una, se asignó la predominante. Para este instrumento la prueba de confiabilidad inter-juez entre dos codificadores fue de un 78\%. Los desacuerdos fueron discutidos y acordados. Los codificadores fueron ciegos al momento de la evaluación.

\section{RESULTADOS}

\subsection{MODELO DE OBSERVACIÓN MEDIADA}

En relación a la primera pregunta de investigación acerca de cómo influir desde la formación inicial en las habilidades de observación y análisis de prácticas pedagógicas en contextos reales, este estudio piloto generó un modelo de uso de la videoteca basado en la observación mediada de prácticas pedagógicas. En lo relativo al uso mediado de la videoteca, la experiencia de este estudio piloto permitió distinguir tres fases importantes a considerar en cada sesión de trabajo: i) una fase de representación en que se presenta el video seleccionado y la transcripción del mismo, ii) una fase de análisis en que a partir de una ficha de lectura en que se presenta un foco de observación se analiza la práctica observada, y iii) una fase de aplicación en que los estudiantes se ven desafiados a transferir lo analizado en la sesión a su experiencia de práctica. La síntesis del modelo para el uso de esta herramienta de práctica virtual se presenta en la Tabla 1.

Tabla 1. Síntesis del modelo de intervención en Formación Inicial

Sesión Modalidad

$1 \quad$ Suspensión de la disposición evaluativa

3
Observación descriptiva

Observación descriptiva e interpretativa
Foco

Ejercitar el paso del juicio a la descripción

Pensamiento del estudiante Pensamiento del estudiante 


\begin{tabular}{l|ll|}
\hline $\mathbf{4}$ & Observación descriptiva e interpretativa & El contenido y su didáctica \\
$\mathbf{5}$ & Observación descriptiva y evaluativa & El contenido y su didáctica \\
$\mathbf{6}$ & Observación descriptiva y evaluativa & Las interacciones de aula \\
$\mathbf{7}$ & Observación descriptiva, interpretativa y evaluativa & Las interacciones de aula \\
$\mathbf{8}$ & Observación descriptiva, interpretativa y evaluativa & Tres focos integrados \\
\cline { 2 - 3 }
\end{tabular}

\subsection{FOCOS DE OBSERVACIÓN}

La segunda pregunta de este estudio apunta a identificar a partir del análisis del protocolo de observación, qué observan los estudiantes, y si la intervención basada en el uso de la videoteca tuvo algún impacto en esta dimensión. En el pre test se observa que en la codificación analítica de cada idea, los estudiantes ponen un mayor foco en el contenido. El elemento del triángulo que menos consideran en sus observaciones es la actividad del estudiante. Este patrón se mantiene en el post test (ver Tabla 2).

Tabla 2. Resultados de focos de observación

\begin{tabular}{lcccc}
\hline & Pre test & \multicolumn{3}{c}{ Post test } \\
\hline & n (ideas) & $\%$ & n (ideas) & $\%$ \\
\hline Estudiante & 13 & $\mathbf{1 8 \%}$ & 18 & $\mathbf{1 7 \%}$ \\
Profesor & 27 & $\mathbf{3 5 \%}$ & 37 & $\mathbf{3 5 \%}$ \\
Contenido & 32 & $\mathbf{4 3 \%}$ & 51 & $\mathbf{4 8 \%}$ \\
Otro & 3 & $\mathbf{4 \%}$ & 1 & $\mathbf{0 \%}$ \\
\hline Total unidad de ideas & 76 & \multicolumn{4}{c}{108} \\
\hline
\end{tabular}

Sin embargo, al analizar el foco de observación a nivel holístico, es decir considerando el texto en forma global y no cada unidad de ideas en forma separada, se observa un aumento de la cantidad de estudiantes que alcanzan los niveles 3 y 4 de integración de los elementos del triángulo instruccional en el post test. De acuerdo a lo que se señaló en la metodología, alcanzar estos niveles supone que los sujetos integran en su registro elementos de la actividad del estudiante. En el pre test un $43 \%$ de los estudiantes alcanzaba los niveles 3 y 4 , en cambio en el post test un $76 \%$ alcanza estos niveles. Esto significa que si bien el estudiante no es foco principal de su observación sí lo incluyen en los registros junto a los demás componentes (ver Tabla 3).

Tabla 3. Resultados de integración de los focos de observación

\begin{tabular}{ccccc} 
& \multicolumn{2}{c}{ Pretest } & \multicolumn{2}{c}{ Post test } \\
\hline Nivel de integración & $\mathrm{n}$ (sujetos) $\%$ & $\mathrm{n}$ (sujetos) & $\%$ \\
\hline 1 & 3 & $\mathbf{1 9 \%}$ & 0 & $\mathbf{0 \%}$ \\
2 & 6 & $\mathbf{3 8 \%}$ & 4 & $\mathbf{2 4 \%}$ \\
3 & 2 & $\mathbf{1 2 \%}$ & 6 & $\mathbf{3 8 \%}$ \\
4 & 5 & $\mathbf{3 1 \%}$ & 6 & $\mathbf{3 8 \%}$ \\
\hline
\end{tabular}




\subsection{MODALIDADES DE OBSERVACIÓN}

Para responder la tercera pregunta de este estudio relacionada con caracterizar cómo observan los estudiantes y analizar el impacto de la intervención en esta dimensión, se analizaron los resultados de la codificación de las modalidades de observación. El esquema de código consideraba 6 patrones de observación, y a cada respuesta se asignó un solo código. El $62 \%$ de los estudiantes en el pre test están agrupados en los niveles 1 y 2 , es decir que presentan un patrón descriptivo. Si bien se codificaron los registros con un código nominal, sustantivamente los niveles más bajos de observación son la descripción general y la evaluación sin descripción. Los estudiantes agrupados en estos dos niveles en el pre test corresponden al $37 \%$ de la muestra, lo que disminuye a un $12 \%$ en el post test. Entre los niveles más altos de observación, la descripción específica se mantuvo en torno a $31 \%$, mientras que la interpretación con descripción subió considerablemente, de $26 \%$ a $50 \%$ de las observaciones (ver Tabla 4).

Tabla 4. Resultados modalidad de observación

\begin{tabular}{lcccc} 
& \multicolumn{2}{c}{ Pretest } & Post test \\
\hline Nivel & $\mathrm{n}$ (sujetos) & $\%$ & $\mathrm{n}$ (sujetos) & $\%$ \\
\hline 1. Descripción general & 5 & $\mathbf{3 1 \%}$ & 1 & $\mathbf{6 \%}$ \\
2. Descripción específica & 5 & $\mathbf{3 1 \%}$ & 5 & $\mathbf{3 2 \%}$ \\
3. Interpretación con descripción & 4 & $\mathbf{2 6 \%}$ & 8 & $\mathbf{5 0 \%}$ \\
4. Evaluación sin descripción & 1 & $\mathbf{6 \%}$ & 1 & $\mathbf{6 \%}$ \\
5. Evaluación con descripción & 0 & $\mathbf{0 \%}$ & 0 & $\mathbf{0 \%}$ \\
6. Descripción, interpretación y evaluación & 1 & $\mathbf{6 \%}$ & 1 & $\mathbf{6 \%}$
\end{tabular}

En la Tabla 5 se presentan ejemplos de textos de los estudiantes en la evaluación inicial y final con el fin de ilustrar los cambios a nivel de foco y modalidad de observación que se han descrito.

Tabla 5. Ejemplos de textos de los estudiantes en el pre y post test

\begin{tabular}{lll}
\hline Respuesta pre test & Respuesta post test & Dimensión \\
\hline Una clase de Matemática & Una clase de mate sobre perímetro. La & QUÉ OBSERVA: \\
dirigida por una profesora & Profesora más 20 alumnos ubicados en & Pasa de alusión a un elemento \\
con distribución de puestos & filas dobles. Empleo de material del triángulo instruccional: \\
en tres filas dobles & concreto lana. Comprender que puede profesor (1 punto), a \\
& haber figuras de diferente forma pero mencionar a los tres \\
& igual contorno. Introducción del componentes (3 puntos), \\
& concepto de perímetro a partir del aunque cabe espacio para \\
& concepto de contorno que ya mayor integración. \\
& manejaban. Explicación del concepto & \\
& científico de la palabra en juego. CÓMO OBSERVA: \\
& Perímetro se vincula con unidades de & Pasa de una descripción \\
& longitud trabajadas con anterioridad. general (1) a una \\
& Figuras distintas pueden tener igual interpretación con \\
& perímetro. & descripción (3). \\
\hline
\end{tabular}


Observé una clase de Observé una clase de matemáticas, en matemática en la cual los alumnos se disponían en filas sentados con compañeros y la profesora estaba frente a la clase.

Observe también que todos los alumnos grabados participaban de la clase ya sea respondiendo las preguntas de la profesora o siguiendo sus instrucciones.

Además, pude apreciar que en las paredes de la sala se encuentran decoradas con motivos de fiestas patrias, lo que se corrobora con la fecha de grabación (23/09) los alumnos al preguntarles a los la que el curso se organizaba con las mesas puestas en columnas de dos filas cada una y la profesora estaba ubicada junto a la pizarra al frente de la clase.

Fui capaz de ver que la profesora recogió los conocimientos previos de alumnos dónde han escuchado el concepto a tratar con anterioridad y para qué es usado en la vida cotidiana, por lo que le da un sentido práctico al concepto.

Se aprecia que diseña una actividad significativa para los niños en la que deben manipular y estar en contacto directo con el concepto a aprender. Se identifica que es sólo al final del video que la profesora relaciona directamente lo aprendido con el subsector de matemática al preguntarles en qué unidad de medida se debe medir el perímetro y les pregunta cuáles son éstas.

\section{QUÉ OBSERVA:}

Pasa de considerar dos elementos (profesora y estudiantes), aunque en forma desarticulada y no relevante para el aprendizaje (2 puntos) a una consideración de los tres elementos en forma integrada (4 puntos).

\section{CÓMO OBSERVA:}

Pasa desde una descripción detallada (2) a una descripción detallada con evaluación e interpretación (7).

\section{DISCUSIÓN}

Este estudio piloto ha permitido explorar el uso de la Videoteca Docente en un contexto de formación. Como primer resultado, cabe destacar que el modelo de observación mediada de videos amplifica las oportunidades de aprendizaje de los estudiantes que estén simultáneamente vinculadas a experiencias de aprendizaje en contextos reales y mediadas intencionalmente por sus programas de FI. Así, el modelo expuesto genera las condiciones para desarrollar habilidades de observación y análisis que permitan a los estudiantes interpretar y evaluar dichas prácticas basándose en evidencia. Como afirman Santagata y Angelici (2010), si bien no puede esperarse que los estudiantes adquieran competencias de expertos, es relevante que los programas de FI hagan visibles las habilidades de observación y reflexión, generando formas probadas para desarrollarlas en sus estudiantes.

Es necesario evaluar las instancias más apropiadas en el currículo de formación para incorporar la tecnología de una plataforma como la Videoteca de Buenas Prácticas Docentes. Esta experiencia piloto se focalizó en una etapa final de la formación (específicamente, en el taller de prácticas), por lo que investigación futura debiera evaluar la integración de la progresión propuesta en el modelo de observación mediada en distintos cursos de la formación, con el fin de favorecer la conexión entre los modelos teóricos correspondientes y las experiencias de práctica en contextos reales. Por otra parte, se hace necesario ampliar el análisis a las alternativas de acción que puedan sugerir 
los estudiantes al observar los videos, ya que este estudio se centró solamente en los focos y modos de observación. Incluir en el modelo una dimensión que implique proyectar la situación de enseñanza observada sugiriendo distintas alternativas de acción puede enriquecer el uso de los videos de clase en la formación docente.

En cuanto a los focos de observación, se pudo constatar que los estudiantes de la muestra tenían un foco de observación centrado en los contenidos. El foco que menos consideraban en sus registros es el estudiante, con un $17 \%$ y $18 \%$ de referencia a este elemento del triángulo instruccional en el pre y post test respectivamente. Esto es especialmente relevante dado que el estudiante es el centro del quehacer profesional de un profesor. En los últimos años se han propuesto varias estrategias, especialmente a nivel de formación continua de profesores y directivos para llevar a los profesores a centrar sus observaciones en los estudiantes (Martinez-Miller y Cervone, 2007; van Es y Sherin, 2008). Si bien no se observaron cambios en la atención de los estudiantes, los resultados sugieren una mayor integración por parte de los participantes de los elementos del triángulo instruccional al final de la intervención. Este resultado indica un avance en las estrategias de observación de los participantes luego de una intervención de tan solo un semestre. Dado que este es un estudio exploratorio, se necesita de estudios con diseños cuasi-experimentales o experimentales, para evaluar estos cambios más apropiadamente.

En relación a la evaluación de la modalidad de observación de los estudiantes, es importante destacar que los esquemas de codificación presentados por la literatura proponen códigos ordinales en que la descripción aparece valorada en el nivel 0, independiente de si es general o específica (Seidel et al., 2011). En el caso de la codificación en este piloto, se tomó la decisión de codificar con códigos nominales, ya que la descripción detallada aparece como una modalidad deseable en tanto se considera la base para una posterior interpretación y evaluación. Dado que el objetivo de esta intervención era favorecer el desarrollo de una modalidad interpretativa o evaluativa, pero basada en evidencia, se consideró como un resultado positivo la disminución de la cantidad de estudiantes que hizo descripciones generales, y el aumento en la cantidad de estudiantes que hizo interpretaciones con descripción.

Finalmente, considerando la experiencia con los supervisores de práctica en este piloto, se sugiere incorporar este modelo de observación mediada al desarrollo profesional de supervisores en los contextos de formación. Los supervisores observan, analizan y retroalimentan las prácticas de los estudiantes, por lo que tener la capacidad para detectar aspectos relevantes de la interacción pedagógica resulta una condición básica para su labor. Por otra parte, el poder analizar en conjunto video clips de prácticas favorece el alineamiento de criterios de supervisión para tener una visión común a la hora de retroalimentar el desempeño de los estudiantes.

\section{REFERENCIAS BIBLIOGRÁFICAS}

Alexander, R. (2008). Culture, Dialogue and Learning: Notes on an Emerging Pedagogy. En N. Mercer (Ed.), Exploring Talk In Schools Inspired By The Work Of Douglas Barnes (pp. 91-114). London: Sage.

Anderson, L. y Krathwohl, D. (Eds.). (2001). A Taxonomy for Learning Teaching, and assessing. A revision of Bloom's taxonomy of educational objectives. New York: Addison Wesley Longman. 
Ball, D.L. y Cohen, D.K. (1999). Developing practice, developing practitioners: Towards a practice-based theory of professional education. En L. Darling-Hammond y G. Sykes (Eds.), Teaching as the learning profession: Handbook of policy and practice (pp. 3-32). San Francisco: Jossey-Bass.

Berliner, D. C. (1994). Expertise: The wonder of exemplary performances. In J. N. Mangiere \& C. C. Block (Eds.), Creating powerful thinking in teachers and students: Diverse perspectives (pp. 161-186). Orlando, FL: Harcourt Brace.

Borko, H., Jacobs, E., Eiteljorg, E. y Pittman, M.E. (2008).Video as a tool for fostering productive discussions in mathematics professional development. Teaching and Teacher Education, vol.24, 417-436.

Borko, H., Koellner, K., Jacobs, J. y Seago, N. (2011). Using video representations of teaching in practice-based professional development programs. Mathematics Education, vol.43, 175-187.

Cohen, D.K., Raudenbush, S. y Ball, D. (2003). Resources, instruction, and research. Educational Evaluation and Policy Analysis, vol.25, n.2, 1-24.

Darling-Hammond, L. y Hammerness, K. (2005). Preparing teachers for a changing world: What teachers should learn and be able to do. United States: Jossey-Bass.

Davis, E. A. (2006). Characterizing productive reflection among preservice elementary teachers: Seeing what matters. Teaching and Teacher Education, vol.22, 281-301.

Echevarria, J. y Short, D. (2004). Using Multiple Perspectives in Observations of Diverse Classrooms. En H. Waxman (Ed.), Observational Research in U.S. Classrooms (pp. 21-71). Cambridge: Cambridge University Press.

Eilam. B. y Poyas, Y. (2006). Promoting awareness of the characteristics of classrooms' complexity: A course curriculum in teacher education. Teaching and Teacher Education, vol.22, 337-351.

Fernández, (2010). Investigating how and what prospective teachers learn through microteaching lesson study. Teaching and Teacher Education, vol.26, n.2, 351362.

Goodwin, C. (1994). Professional Vision. American Anthropologist, vol.96, n.3, 606-633.

Grossman, P. y Mc Donald, M. (2008). Back to the Future: Directions for Research in Teaching and Teacher Education. American Educational Research Journal, vol.45, n.1, 184-205.

Hammer, D. y Van Zee, E. (2006). Seeing the Science in Children's Thinking: Case Studies of Student Inquiry in Physical Science. A Staff Developer's Guide. Heinemann Portsmouth: NH.

Hiebert, J., y Stigler, J. W. (2000). A proposal for improving classroom teaching: Lessons from the TIMSS Video Study. Elementary School Journal, vol.101, n.1, 3-20.

Koc, Y., Peker, D. y Osmanoglu, A. (2009). Supporting teacher professional development through online video case study discussions: An assemblage of preservice and inservice teachers and the case teacher. Teaching and Teacher Education, vol.25, 1158-1168.

Kucan, L., Sullivan, A., Khasnabais, D. y Chang, C. (2009). The Video Viewing Task: A source of information for assessing and addressing teacher understanding of textbased discussion. Teaching and Teacher Education, vol.25, 415-423.

Lewis, C. (2002). Does lesson study have a future in the United States? Nagoya journal of education and human development, vol.1, n.1, 1-23. 
Manzi, J., González, R. y Sun, Y. (Eds.) (2001). La Evaluación Docente en Chile. Santiago de Chile: Mide UC.

Martinez-Miller, P. y Cervone, L. (2007). Breaking Through to Effective Teaching: A Walk-Through Protocol Linking Student Learning and Professional Practice. Los Angeles: UCLA School Management Program.

Milicic, N., Rosas, R., Scharager, J., García, M. y Godoy, C. (2008). Diseño y construcción y evaluación de una pauta de observación de videos para evaluar calidad del desempeño docente. Psykhe, vol.17, n.2, 79-90.

Perry, R., y Lewis, C. (2009). What is successful adaptation of lesson study in the US? Journal of Educational Change, vol.10, 365-391.

Preiss, D.D. (2009). The Chilean instructional pattern for the teaching of language: a video-survey study based on a national program for the assessment of teaching. Learning and Individual Differences, vol.19, 1-11.

Preiss, D.D. (2010). Folk Pedagogy and Cultural Markers in Teaching: Three Illustrations from Chile. In D.D. Preiss y R.J. Sternberg (Eds.), Innovations in Educational Psychology: Perspectives on Learning, Teaching, and Human Development (pp. 325-356). New York: Springer Publishing Company.

Preiss, D.D., Larraín, A. y Valenzuela, S. (2011). Discurso y pensamiento en el aula matemática chilena. Psykhe, n. 20, 17-32.

Preiss, D.D. y Sternberg, R.J. (Eds.). (2010). Innovations in Educational Psychology: Perspectives on teaching, learning and human development. New York: Springer Publishing Company.

Radovic, D. y Preiss, D.D. (2010). Patrones de Discurso Observados en el Aula de Matemática de Segundo Ciclo Básico en Chile. Psykhe, vol.19, 65-79.

Santagata, R. y Angelici, G. (2010). Studying the Impact of the Lesson Analysis Framework on Pre-service Teachers' Abilities to Reflect on Videos of Classroom. Teaching Journal of Teacher Education, vol.61, n.4, 339-349.

Schwartz, D. y Hartman, K. (2007). It's not Video Anymore: Designing Digital Video for Learning and Assessment. En R. Goldman et al. (Eds.), Video Research in the learning sciences (pp. 335-348). Mahwah, N.J.: Lawrence Erlbaum Associates.

Seidel, T., Strümmer, K., Blomberg, G., Kobarg. y Schwindt, K. (2011). Teachers learning from analysis of videotaped classroom situations: Does it make a difference whether teachers observe their own teaching or that of others? Teaching and Teacher Education, vol.27, 259-267.

Sherin, M. (2007). The development of Teacher's Professional Vision in Video Clubs. En R. Goldman et al. (Eds.) Video Research in the learning sciences (pp. 383-396). Mahwah,N.J.: Lawrence Erlbaum Associates.

Sherin, M. y Han, S. (2004). Teacher learning in the context of a video club. Teaching and Teacher Education, vol.20, 163-183.

Sherin, M. y van Es, E. (2009). Effects of Video Club Participation on Teachers' Professional Vision. Journal of Teacher Education, vol.60, n.1, 20-37.

Sims, L. y Walsh, D. (2009). Lesson Study with pre-service teachers: Lessons from lessons. Teaching and Teacher Education, vol.25, 724-733. 
Sternberg, R.J. y Grigorenko, E.L. (2003). Teaching for successful intelligence: Principles, procedures, and practices. Journal for the Education of the Gifted, vol.27, n.2-3, 207-228.

Sternberg, R.J. y Horvath, J.A. (1995). A prototype view of expert teaching. Educational Researcher, vol.24, n.6, 9-17.

Tochon, F. (2007). From video cases to video pedagogy: a framework for video feedback and reflection in pedagogical research praxis. En R. Goldman et al. (Eds.), Video Research in the learning sciences (pp. 53-66). Mahwah, N.J.: Lawrence Erlbaum Associates.

van Es, E. y Sherin, M. (2008). Mathematics teachers" "learning to notice" in the context of a video club. Teaching and Teacher Education, vol.24, 244-276.

Zhang, M., Lundeberg, M., Köhler M. y Eberhardt, J. (2011). Understanding the affordances and challenges of three types of video for teacher professional development. Teaching and Teacher Education, vol.27, 454-462. 